\title{
Systemic delivery of large-scale manufactured Wharton's Jelly mesenchymal stem cell-derived extracellular vesicles improves cardiac function after myocardial infarction
}

\author{
Michael A. Bellio ${ }^{1, \#}$, Rosemeire M. Kanashiro-Takeuchi ${ }^{1,2, \#}$, Lauro Takeuchi ${ }^{1}$, Shathiyah Kulandavelu ${ }^{1,3}$, Yee- \\ Shuan Lee ${ }^{1}$, Wayne Balkan ${ }^{1,4}$, Karen C. Young ${ }^{1,5}$, Joshua M. Hare ${ }^{1,4}$, Aisha Khan ${ }^{1}$ \\ 'Interdisciplinary Stem Cell Institute, University of Miami Miller School of Medicine, Miami, FL 33136, USA. \\ 2Departments of Molecular and Cellular Pharmacology, University of Miami Miller School of Medicine, Miami, FL 33136, USA. \\ ${ }^{3}$ Departments of Pediatrics, University of Miami Miller School of Medicine, Miami, FL 33136, USA. \\ ${ }^{4}$ Departments of Medicine, University of Miami Miller School of Medicine, Miami, FL 33136, USA. \\ ${ }^{5}$ Division of Neonatology, University of Miami Miller School of Medicine, Miami, FL 33136, USA. \\ \#Authors contributed equally.
}

Correspondence to: Dr. Aisha Khan, Interdisciplinary Stem Cell Institute, University of Miami Miller School of Medicine, 1501 NW 10th Ave, Miami, FL 33136, USA. E-mail: AKhan@med.miami.edu

\begin{abstract}
How to cite this article: Bellio MA, Kanashiro-Takeuchi RM, Takeuchi L, Kulandavelu S, Lee YS, Balkan W, Young KC, Hare JM, Khan A. Systemic delivery of large-scale manufactured Wharton's Jelly mesenchymal stem cell-derived extracellular vesicles improves cardiac function after myocardial infarction. J Cardiovasc Aging 2022;2:9. https://dx.doi.org/10.20517/jca.2021.21
\end{abstract}

Received: 9 Sep 2021 First decision: 23 Sep 2021 Revised: 18 Oct 2021 Accepted: 26 Oct 2021 Published: 5 Jan 2022

Academic Editors: AJ Marian, Roberto Bolli Copy Editor: Xi-Jun Chen Production Editor: Xi-Jun Chen

\begin{abstract}
Introduction: Cardiovascular disease and myocardial infarction are leading causes of morbidity and mortality in aged populations. Mesenchymal stem cell (MSC)-derived extracellular vesicles (EVs) are under evaluation as a therapeutic option for the treatment of myocardial infarction.
\end{abstract}

Aim: This study aimed to develop a large-scale manufacturing procedure to harvest clinical-grade EVs required for the translation of EVs to the clinic.

Methods and Results: We compared the efficiency of large scale MSC-derived EV production and characterized EV miRNA cargo using the Quantum bioreactor with either fetal bovine serum or human platelet lysate (PLT)containing expansion media. We tested the potency of the EV products in a murine model of acute myocardial infarction. Our results demonstrate an advantage of the Quantum bioreactor as a large-scale platform for EV 
production using PLT media; however, both media produced EVs with similar effects in vivo. The systemic delivery of EV products improved cardiac function following myocardial infarctions as indicated by a significant improvement in ejection fraction as well as parameters of cardiac performance, afterload, contractility and lusitropy.

Conclusion: These findings have important implications for scale-up strategies of EVs and will facilitate clinical trials for their clinical evaluation.

One sentence summary: Large scale manufacturing of MSC-derived EVs is feasible and when delivered systemically, improves cardiac function after myocardial infarction.

Keywords: Extracellular vesicles, Wharton's Jelly, mesenchymal stem cells, manufacturing, myocardial infarction

\section{INTRODUCTION}

Cardiovascular aging is associated with molecular and physiological changes that result in the increased incidence of cardiovascular diseases such as hypertension, atherosclerosis, and myocardial infarction $(\mathrm{MI})^{[1]}$. As the aged population continues to grow in number, novel therapies aimed at repairing the damaged cardiovascular system are required to provide effective medical solutions. Mesenchymal stem cell (MSC) derived extracellular vesicles (EVs) are presently under evaluation as a potential regenerative medicine approach. MSCs are found in many human organs and have been safely tested in clinical trials using an allogeneic strategy ${ }^{[2-4]}$. Phase I/II clinical trials such as POSEIDON ${ }^{[5]}$, POSEIDON-DCM $^{[6]}$, and TRIDENT $^{[7]}$ have demonstrated the safety and potential efficacy of allogeneic MSCs for the treatment of ischemic and non-ischemic cardiomyopathy. In terms of mechanism of action, there is growing awareness that MSCs release EVs and other growth factors that exert paracrine effects. Given the ability to purify EVs, it seems logical to test them for their ability to exert many of the actions of the parent cells ${ }^{[s, 9]}$.

Pre-clinical data collected across multiple regenerative medicine models suggests that a primary mechanism of MSC-induced regeneration is mediated by the release of EVs into the extracellular space ${ }^{[10,11]}$. EVs are important for cell-to-cell communication and produce a paracrine effect when used as a therapeutic agent. MSC-derived EVs promote tissue recovery through restoring tissue homeostasis, inhibiting inflammation, and promoting angiogenesis ${ }^{[12-15]}$. Isolation of MSC-derived EVs from in vitro conditioned media has demonstrated important cardiac reparative effects, and thus further exploration is warranted ${ }^{[16]}$.

Despite promising pre-clinical data, several challenges impede the translation of EVs into cardiovascular clinics. These challenges include establishing advanced characterization methods for documented reproducibility, the large-scale production of EV concentrated fluid, and the large-scale isolation and processing of clinical-grade, FDA-compliant EV products for Investigational New Drug enabling studies or clinical application $^{[17]}$. Traditional EV production techniques are often limited due to the need for repeated and lengthy manufacturing protocols and characterization of each product lot. Furthermore, the need for repetitive lot productions of cell source products can lead to greater batch-to-batch variability and the need for repeated manufacturing runs and testing time.

Wharton's Jelly (WJ), derived from umbilical cord tissue, is a good source of MSCs due to non-invasive collection techniques and the natural pool of fetal sourced cell types. Therefore, we developed a large-scale manufacturing protocol utilizing Wharton's Jelly-derived MSCs (WJMSCs) to efficiently streamline the manufacture of EVs for research and clinical application. 
During the development of our large-scale production methodology, we investigated the effect of cell culture media composition on the efficiency and characteristics of EV production. Fetal bovine serum (FBS) is commonly used as a growth media supplement for the expansion of $\mathrm{MSCs}^{[18]}$. However, due to the FDA's concerns involving the use of animal-derived products, human platelet lysate (PLT) has become a commonly used alternative for clinical cell expansion. PLT is a safe and effective alternative to FBS that retains MSC phenotype and promotes cell proliferation and expansion potential ${ }^{[19]}$.

MSC-derived EVs have a well-documented track record of promoting cardiac protection and restoring cardiac function in various models of $\mathrm{MI}^{[20]}$. In these models, EVs are administered immediately after cardiac injury and administered by intra-myocardial injection or by tail vein ${ }^{[2,22]}$. The immediate delivery of EVs to an injury site speaks to the cardioprotective effects of MSC-derived EVs and the therapeutic value of reducing or preventing the initial damage caused by MI. However, these models have yet to explore EVs as a therapeutic option after established MI injury and the subsequent long-term effects that the EV products have on cardiac function.

In this study, we used manufacturing data and in vivo analysis to determine the optimal conditions to produce large quantities of EVs for use as a therapeutic for MI. We hypothesized that PLT-supplemented media would be superior for the large-scale expansion and production of EVs and that systemic delivery of WJMSC-derived EVs would improve cardiac function and reduce cardiac remodeling compared to placebo.

\section{METHODS}

\section{WJMSC isolation and expansion}

This study protocol and written informed consent were reviewed and approved by the Institutional Review Board of the University of Miami (IRB number 20100986). WJ was isolated from human umbilical cord obtained from healthy and full-term infants. WJ was cut into small pieces, placed into several Petri dishes with a minimum medium to allow for attachment. The dishes were placed into a $37^{\circ} \mathrm{C}$ incubator with $5 \%$ $\mathrm{CO}_{2}$. The growth medium was composed of $20 \% \mathrm{FBS}$ and $1 \%$ penicillin/streptomycin in $\alpha$-MEM or $5 \%$ PLT max (Millcreek), heparin, and 1\% penicillin/streptomycin in $\alpha$-MEM. Three umbilical cords were used for these experiments, where each cord was split into FBS and PLT culture after WJ isolation. One day after plating, a small amount of medium was added to the WJ to ensure the WJ pieces were attached securely. The medium was replenished every three to four days until the colonies were $90 \%$ confluent. Once confluent, the cells were rinsed once with phosphate-buffered saline (PBS) and then incubated with TrypLE (Thermofisher) for $5 \mathrm{~min}$ at room temperature, and the TrypLE was neutralized with medium and the cell suspension centrifuged at $200 \mathrm{xg}$ for $10 \mathrm{~min}$. The cells were counted, and 0.5 to $1 \times 10^{6}$ cells were seeded into each T-175 flask. The cells in these new flasks were designated as passage 1. Fresh medium was replenished every three to four days until the cells reached $90 \%$ confluency. When passage 1 cells reached confluency, the cells were harvested and cryopreserved at 2 to $5 \times 10^{6}$ cells $/ \mathrm{mL}$ of cryopreservation medium. The cryopreservation medium used was CryoStor CS10 (BioLife Solutions). Flow Cytometry analysis with CD31, CD45, CD105, CD90, and isotype control was performed on P2 WJMSC expanded in both PLT and FBS to confirm their MSC origin [Supplementary Figure 1].

\section{Quantum bioreactor MSC expansion and EV isolation}

The Quantum bioreactors (Terumo BCT) were primed with PBS and coated with $5 \mathrm{mg}$ of human fibronectin (Corning) diluted in $100 \mathrm{~mL}$ of PBS. Fibronectin coating was performed for a minimum of $4 \mathrm{~h}$, followed by a systemic washout with expansion media supplement with either $20 \%$ FBS or $5 \%$ PLT. After expansion media exchange, $30.0 \times 10^{6}$ WJMSCs were loaded and allowed to attach for $24 \mathrm{~h}$. Once cell attachment was completed, cell expansion was performed by increasing the daily media input feeding rate 
to compensate for the growing number of cells. Samples of the outer loop media were collected daily to test for lactate production, which was used to estimate the total number of growing cells. The estimation and prediction of cell numbers were determined by daily lactate production readings, media flow rate, and excel sheet calculations and formulas provided by Terumo BCT. After peak expansion was obtained, expansion media was washed away with inner and outer loop washouts with PBS. After completion of the washout cycle, the media was replaced with $\alpha$-MEM without FBS or PLT. Therefore, EV manufacturing was performed without the presence of any protein supplement. Media conditioning was then allowed to continue for $120 \mathrm{~h}$, and inner loop outlet waste media was collected daily for EV isolation. Every $24 \mathrm{~h}$, $200 \mathrm{~mL}$ of conditioned media was collected from the Quantum bioreactor and subjected to sequential centrifugation at $2000 \mathrm{xg}$ for $30 \mathrm{~min}$ followed by $20,000 \mathrm{xg}$ for $30 \mathrm{~min}$ to remove large EVs prior to ultracentrifugation. Ultracentrifugation (Beckman Coulter) was performed at 100,000 $\mathrm{xg}$ for $70 \mathrm{~min}$ using a Tizo rotor to pellet the remaining EVs. EV pellets were resuspended in 4-5 $\mathrm{mL}$ of saline and frozen at $-80{ }^{\circ} \mathrm{C}$. Samples were resuspended in sterile saline to make the final product ready for injection.

\section{Nanosight analysis}

Nanosight nanoparticle analysis was performed on the final EV products using the Nanosight NS300 instrument (Malvern Panalaytical) and NTA 3.3 Dev Build 3.3.104 software. Mean concentrations and mode size were determined from 5 videos taken of 1 sample analyzed at a 1:1000 dilution, pump speed 30, video length $30 \mathrm{~s}$.

\section{EV flow cytometry analysis}

Flow cytometry of EV nanoparticles was completed using Exosome-Human CD63 Isolation/Detection Reagent (Thermofisher) according to the manufacturer's instructions. EVs collected at $48 \mathrm{~h}$ and $120 \mathrm{~h}$ were analyzed as representative examples for the detection of CD63 and CD81, exosome surface markers. Precipitated CD63 particles were stained with CD63 antibody, CD81 antibody, or FITC and APC isotype controls. Samples were analyzed on a Cytoflex flow cytometer (Beckman Coulter).

\section{miRNA isolation, sequencing, and bioinformatics}

WJMSC-derived EVs isolated from three donors were manufactured from FBS- and PLT-expanded cells, and processed for miRNA isolation and sequencing in triplicate. miRNA extraction and sequencing were conducted by QIAGEN Genomic Services. RNA was isolated using the exoRNeasy Kit Part II (Qiagen) and automated on the QIAcube. $700 \mu \mathrm{L}$ of QIAzol was added to the EVs and then continued with the 2nd part of the exoRNeasy midi kit for RNA purification as per manufacturer's protocol. RNA was extracted from the samples, including RNA spike-ins in the purification step to allow monitoring of the RNA extraction efficiency. Each RNA sample was successfully reverse transcribed (RT) into cDNA and tested for the expression of 5 miRNAs and three synthetic spike-ins. RNA was reversely transcribed in $10 \mu \mathrm{L}$ reactions using the miRCURY LNA RT Kit (Qiagen). cDNA was diluted 100× and assayed in $10 \mu \mathrm{L}$ PCR reactions using the miRCURY LNA SYBR Green PCR Kit (Qiagen). The amplification was performed in a LightCycler 480 System (Roche) in 384-well plates. The amplification curves were analyzed using Roche LC software, both for determination of $\mathrm{Cq}$ (by the 2 nd derivative method) and for melting curve analysis. The raw data was extracted from the Roche LC software. The evaluation of expression levels was performed based on raw Cq values. The library preparation was done using the QIAseq miRNA Library Kit (QIAGEN). A total of $5 \mu \mathrm{L}$ total RNA was converted into miRNA NGS libraries. Adapters containing unique molecular identifiers were ligated to the RNA. Then RNA was converted to cDNA. The cDNA was amplified using PCR (22 cycles), and during the PCR, indices were added. After PCR, the samples were purified. Library preparation QC was performed using either Bioanalyzer 2100 (Agilent) or TapeStation 4200 (Agilent). The library pool was then sequenced on a NextSeq500 sequencing instrument according to the manufacturer's instructions. Raw data was de-multiplexed, and FASTQ files for each sample were 
generated using the bcl2fastq software (Illumina Inc.). FASTQ data were checked using the FastQC tool. FASTQ data was processed in QIAGEN's Omicsoft Array Suite (version 10.1.1.14). Following the preprocessing of the reads, reads were aligned to the genome using OSA. All statistical analysis and miRNA identification was performed by the QIAGEN Genomic Services. Bioinformatics was completed using the IPA Knowledge Base software (version 01-16), and mature miRNA and mRNA target relationships were determined. All gene targeting was limited to relationships with experimental observed findings. mRNA target lists were used for Bioprofiler analysis to identify myocardial infarction associated genes. Vinny 2.1 was used to create vin diagram comparison charts.

\section{Murine myocardial infarction model and EV treatment}

All animal experiments were performed in accordance with the protocol approved by the University of Miami IACUC. Three-month-old male C57BL6J mice were induced and anesthetized with isoflurane (2\%$3 \%$ ) inhalation. Electrocardiogram, body temperature, and heart rate were monitored throughout the procedure. MI was induced through the permanent ligation of the left coronary artery with a 7-0 Prolene suture as previously described with minor modifications ${ }^{[23]}$. MI was confirmed by ST elevation and myocardium blanching during the procedure. Extended-release buprenorphine was given pre-operatively $(0.5-1 \mathrm{mg} / \mathrm{kg})$. Echocardiography at day 7 , post-surgery was performed before randomization to one of three treatment groups: placebo $(n=14)$, EV-PLT (EVs isolated in serum-free media from PLT-expanded WJMSCs, $n=14$ ), or EV-FBS (EVs isolated in serum-free media from FBS-expanded WJMSCs, $n=10$ ).

EV products were delivered by intra-jugular injection at a dose of $1 \times 10^{10}$ particles in $100 \mu \mathrm{L}$ of saline, while $100 \mu \mathrm{L}$ of saline only was used as the placebo. Treatments began 1 week after the MI and were repeated weekly; the animals received 6 total doses of EVs or placebo and were monitored for 8 weeks [Supplementary Figure 2]. To perform the weekly injections, the animals were anesthetized with isoflurane $(1 \%-3 \%)$, and the area over the right and/or left jugular was shaved to expose the external jugular veins. The $29 \mathrm{G}$ needle (BD) was inserted through the pectoral muscle below the sternoclavicular junction. After the injection, gentle pressure to the injection site was applied to prevent bleeding, and the animal was allowed to recover before its return to the cage. Investigators administering the products were blinded to the treatment.

\section{Echocardiography}

Cardiac function [EF, cardiac output (CO), and stroke volume (SV)] were calculated using Vevo-2100 imaging system (Visual Sonics Inc.) at baseline, 1-, 4- and 8-week post-MI as previously described with minor modifications ${ }^{[23,24]}$. Briefly, mice were anesthetized by inhalation with $1 \%-3 \%$ isoflurane while heart rates and body temperatures were continuously monitored. Cardiac morphology and function were analyzed using an automated left ventricular (LV) analysis software (AutoLV, Visual Sonics Inc.) at baseline, 1-, 4-, and 8-week post-MI. All assessments of imaging and data analysis were performed by the investigator blinded to the treatment.

\section{Hemodynamic study}

Pressure-volume data were obtained from steady-state and inferior vena cava occlusion using a Power Lab data acquisition system (ADInstruments, Denver, CO) as previously described ${ }^{[23,24]}$. A microtip pressurevolume catheter (SPR 839, Millar Instruments) was introduced into the surgically exposed right carotid artery and advanced into the left ventricle. Hemodynamic data were analyzed offline by investigators blinded to treatment groups using Lab Chart 8.1.5 Pro Software (ADInstruments). The volume calibration was performed using end-diastolic volume and end-systolic volume derived from echocardiographic measurements. 


\section{MI quantification}

Masson's trichrome-stained sections were used to assess infarct size. Histological sections were scanned with a digital pathology slide scanner (PathScan Enabler IV, Meyer Instruments), and infarct size was measured using MIQuant software as described ${ }^{[25]}$. The infarcted LV wall was calculated by the midline length measurement (calculated by dividing the midline length of the infarcted LV wall by the midline length of the total LV wall) as previously validated by Nascimento et al. ${ }^{[25]}$. Only regions with infarct in > $50 \%$ of the whole thickness of the myocardium were considered for infarct midline ${ }^{[26]}$.

\section{Statistics}

Statistics were completed using Prism 8 (Version 8.4.2). WJMSC EV expansion data was analyzed betweenand within-group using two-way ANOVA and multiple comparisons test with Tukey. $P$-values less than 0.05 were significant. Two group column statistics were performed using a 2 -tail $t$-test. Echocardiography and pressure-volume (PV) loop data were analyzed using one-way ANOVA with Tukey's multiple comparison test. Infarct size was analyzed by one-way ANOVA comparing the mean of each group to placebo with Dunnett multiple comparison testing. Averaged data with error bars include \pm the standard error of the mean.

\section{RESULTS}

\section{Large scale expansion efficiently produced WJMSC-derived EVs in both FBS and PLT}

WJMSC expansion in the Quantum bioreactors was completed in six to seven days. Throughout this expansion time, daily lactate measurements were recorded to assess cell growth. Maximum lactate production was achieved after six days of expansion, indicating peak cell confluency. Comparative analysis of lactate production and the calculated predicted cell yield between PLT or FBS demonstrated a more efficient cell expansion of WJMSC when expanded in media with PLT. Significantly higher lactate measurements in the PLT expansion at day $6(16.0 \pm 1.6 \mathrm{mmol} /$ day in PLT vs. $7.43 \pm 1.7 \mathrm{mmol} /$ day in FBS, $P$-value $<0.001)$ indicates a superior cell expansion [Figure 1A]. The predicted cell number, which is based on daily lactate production, further demonstrated the improved cell expansion using the PLT media compared to FBS [Figure 1B].

After maximum cell expansion was obtained, PLT and FBS containing medias were replaced with serumfree media, and EV harvest and isolation were performed every $24 \mathrm{~h}$ for $120 \mathrm{~h}$. No significant difference was observed in the number of particles harvested daily in the FBS or PLT groups [Figure 1C]. Similarly, there was no significant difference between the total number of harvested particles, $1.64 \pm 0.2 \times 10^{13}$ and $1.13 \pm 0.5 \times 10^{13}$ nanoparticles for the PLT and FBS group, respectively [Figure 1D]. Furthermore, nanoparticle tracking analysis of the harvested products revealed no difference in the mode particle size of the EVs from each group, an average mode size of $125 \pm 4.4 \mathrm{~nm}$ and $113 \pm 6.2 \mathrm{~nm}$ for the PLT and FBS group, respectively [Figure $1 \mathrm{E}$ and $\mathrm{F}$ ].

EV products collected at 48 and $120 \mathrm{~h}$ were analyzed by flow cytometry to confirm the presence of EV markers CD63 and CD81. There was no observed difference in the expression of these exosome markers between FBS- and PLT-expanded products [Figure 2].

\section{Comparative analysis of FBS vs. PLT produced EV cargo}

Sequencing of the miRNA cargo was completed using the three-donor manufactured WJMSC-EVs in triplicate. Results revealed 157 mature miRNAs in PLT-EVs and 154 mature miRNAs in FBS-EVs with greater than 100 copies [Figure 3A and B, Supplementary Table 1]. Direct comparison between the two groups showed 142 commonly expressed miRNAs, 15 unique miRNAs in the PLT group, and 12 unique miRNAs in the FBS group [Figure 3C, Supplementary Table 2]. Qiagen IPA bioinformatics analysis 

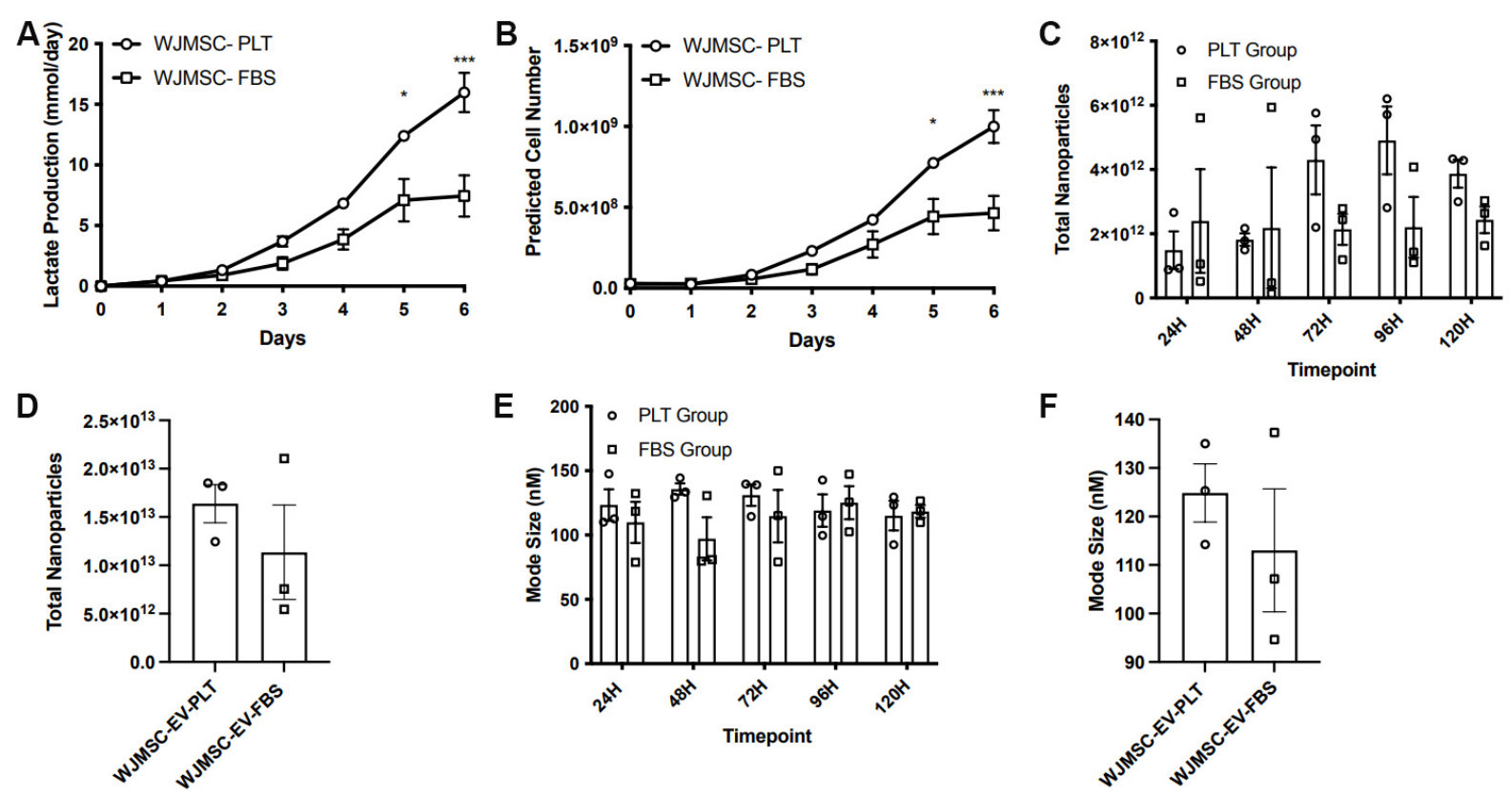

Figure 1. WJMSC expansion and EV manufacturing in the quantum bioreactor. (A) Recorded values of the daily lactate production during WJMSC expansion. (B) The daily prediction of cell numbers within the bioreactor. (C) The average number of EVs isolated at each collection point. (D) The average total number of EVs collected in a single bioreactor run. (E) The average mode size of the EV harvest at each collection time point. (F) The average mode size of the EVs isolated from a single bioreactor run. ${ }^{\star} P<0.05,{ }^{\star \star \star} P<$ 0.001. WJMSC: Wharton's Jelly-derived mesenchymal stem cell; EVs: extracellular vesicles; PLT: human platelet lysate; FBS: fetal bovine serum.

connected 68 miRNAs to 1024 mRNA targets in the PLT group and 64 miRNAs to 1048 mRNA in the FBS group. Comparison of these mRNA targets showed 993 common mRNA targets with 28 unique targets in the PLT group and 52 unique targets in the FBS group [Figure 3D, Supplementary Tables 3 and 4]. Analysis of miRNA targets was completed to identify gene targets associated with MI [Figure 4].

\section{Evaluation of EV administration post-MI by echocardiography}

Cardiac function was assessed in adult male mice by echocardiography at baseline and after MI at weeks 1, 4, and 8. One week after myocardial injury, mice were randomized into placebo and EV treatment groups. Re-evaluation of cardiac function at 4-week showed no significant changes in the EV treated group compared to placebo. However, significant improvement in EF was evident at 8-week in both the WJMSCEV-PLT and WJMSC-EV-FBS groups vs. placebo [Figure 5A, Table 1]. Similarly, EV administration significantly elevated CO after 8-week compared to placebo [Figure 5B, Table 1] and significantly rescued SV [Figure 5C, Table 1]. No significant changes in fractional shortening, cardiac volume, or area parameters were observed [Table 1]. Heart rate, body weight, and other cardiac structure parameters were unaffected by the treatment [Table 1].

\section{Integrated hemodynamic responses to EV treatment post MI}

Left ventricular micromanometry catheterization was performed 7-week after the first EV administration to assess hemodynamic parameters within each group [Figure 6, Table 2]. Administration of both EV preparations similarly improved cardiac parameters of integrated performance, afterload, contractility, and lusitropy. Integrated performance parameters found to be increased in both EV groups include stroke work [Figure 7A, Table 2], CO [Figure 7B, Table 2], SV [Figure 7C, Table 2], and EF [Figure 7D, Table 2]. At the same time, the arterial-ventricular coupling ratio (Ea/Ees) was not different among groups [Table 2]. Afterload, indexed by arterial elastance (Ea), was significantly reduced in both EV treatment groups 
Table 1. Echocardiography measurements of cardiac function parameters

\begin{tabular}{|c|c|c|c|c|}
\hline & Time point & Placebo & WJMSC-EV-PLT & WJMSC-EV-FBS \\
\hline \multirow[t]{4}{*}{ Ejection fraction (\%) } & Baseline & $40.1 \pm 1.9$ & $44.7 \pm 3.2$ & $44.3 \pm 2.7$ \\
\hline & Week 1 & $15.4 \pm 1.9$ & $16.9 \pm 1.9$ & $17.3 \pm 3.0$ \\
\hline & Week 4 & $17.1 \pm 1.8$ & $19.5 \pm 1.7$ & $16.4 \pm 2.8$ \\
\hline & Week 8 & $11.8 \pm 1.4$ & $21.9 \pm 2.3^{\star \star}$ & $21.9 \pm 3.5^{\star}$ \\
\hline \multirow[t]{4}{*}{ Cardiac output ( $\mu \mathrm{L}$ ) } & Baseline & $10.4 \pm 0.5$ & $12.0 \pm 0.6$ & $10.2 \pm 0.6$ \\
\hline & Week 1 & $8.06 \pm 1.0$ & $8.09 \pm 0.8$ & $8.30 \pm 0.9$ \\
\hline & Week 4 & $11.1 \pm 0.9$ & $10.9 \pm 1.0$ & $8.70 \pm 0.9$ \\
\hline & Week 8 & $8.70 \pm 0.9$ & $15.2 \pm 1.2^{\star \star \star}$ & $13.2 \pm 1.0^{\star}$ \\
\hline \multirow[t]{4}{*}{ Stroke volume $(\mu \mathrm{L})$} & Baseline & $21.4 \pm 1.1$ & $23.9 \pm 1.4$ & $20.6 \pm 1.3$ \\
\hline & Week 1 & $15.4 \pm 1.9$ & $15.1 \pm 1.3$ & $15.8 \pm 1.8$ \\
\hline & Week 4 & $21.4 \pm 1.8$ & $22.5 \pm 2.1$ & $16.9 \pm 1.5$ \\
\hline & Week 8 & $16.7 \pm 1.6$ & $27.9 \pm 2.0^{\star \star \star}$ & $26.0 \pm 2.3^{\star}$ \\
\hline \multirow[t]{4}{*}{ Fractional shortening (\%) } & Baseline & $7.74 \pm 0.6$ & $8.82 \pm 0.7$ & $7.06 \pm 0.6$ \\
\hline & Week 1 & $4.55 \pm 0.6$ & $3.15 \pm 0.3$ & $4.61 \pm 0.6$ \\
\hline & Week 4 & $5.12 \pm 0.8$ & $3.98 \pm 0.5$ & $4.01 \pm 0.6$ \\
\hline & Week 8 & $2.67 \pm 0.6$ & $4.21 \pm 0.5$ & $4.59 \pm 0.5$ \\
\hline \multirow[t]{4}{*}{ Volume $(\mu \mathrm{L})$} & Baseline & $51.5 \pm 2.5$ & $51.2 \pm 3.5$ & $42.7 \pm 3.0$ \\
\hline & Week 1 & $105.7 \pm 8.6$ & $94.6 \pm 9.4$ & $94.0 \pm 7.8$ \\
\hline & Week 4 & $122.5 \pm 9.6$ & $115.0 \pm 13.6$ & $109.7 \pm 10.1$ \\
\hline & Week 8 & $140.0 \pm 15.2$ & $131.5 \pm 14.5$ & $120.0 \pm 13.8$ \\
\hline \multirow[t]{4}{*}{ Volume-diastolic $(\mu \mathrm{L})$} & Baseline & $53.3 \pm 1.2$ & $55.3 \pm 3.0$ & $46.7 \pm 1.5$ \\
\hline & Week 1 & $110.4 \pm 8.5$ & $95.4 \pm 8.6$ & $99.2 \pm 7.4$ \\
\hline & Week 4 & $135.0 \pm 10.8$ & $124.3 \pm 14.9$ & $115.7 \pm 9.1$ \\
\hline & Week 8 & $156.6 \pm 15.4$ & $142.7 \pm 14.6$ & $132.5 \pm 11.7$ \\
\hline \multirow[t]{4}{*}{ Volume-systolic $(\mu \mathrm{L})$} & Baseline & $31.9 \pm 1.3$ & $31.3 \pm 3.0$ & $26.1 \pm 1.7$ \\
\hline & Week 1 & $95.0 \pm 8.0$ & $80.2 \pm 8.4$ & $83.4 \pm 8.0$ \\
\hline & Week 4 & $113.5 \pm 10.5$ & $101.8 \pm 13.9$ & $98.8 \pm 10.0$ \\
\hline & Week 8 & $140.0 \pm 15.2$ & $114.7 \pm 14.8$ & $106.5 \pm 12.7$ \\
\hline \multirow[t]{3}{*}{ Area $\left(\mathrm{mm}^{2}\right)$} & Baseline & $20.4 \pm 0.7$ & $20.4 \pm 0.8$ & $18.2 \pm 0.8$ \\
\hline & Week 1 & $30.9 \pm 1.6$ & $28.6 \pm 1.7$ & $28.9 \pm 1.4$ \\
\hline & Week 4 & $33.7 \pm 1.6$ & $32.2 \pm 2.2$ & $31.5 \pm 1.7$ \\
\hline
\end{tabular}




\begin{tabular}{|c|c|c|c|c|}
\hline & Week 8 & $37.5 \pm 2.3$ & $35.4 \pm 2.3$ & $32.9 \pm 2.3$ \\
\hline \multirow[t]{4}{*}{ Area-diastolic $\left(\mathrm{mm}^{2}\right)$} & Baseline & $21.0 \pm 0.3$ & $21.5 \pm 0.7$ & $19.3 \pm 0.3$ \\
\hline & Week 1 & $31.8 \pm 1.5$ & $29.0 \pm 1.6$ & $29.9 \pm 1.4$ \\
\hline & Week 4 & $35.9 \pm 1.6$ & $33.7 \pm 2.2$ & $32.6 \pm 1.5$ \\
\hline & Week 8 & $39.0 \pm 2.3$ & $37.1 \pm 2.2$ & $35.4 \pm 1.8$ \\
\hline \multirow[t]{4}{*}{ Area-systolic $\left(\mathrm{mm}^{2}\right)$} & Baseline & $15.5 \pm 0.4$ & $15.3 \pm 0.9$ & $13.8 \pm 0.4$ \\
\hline & Week 1 & $28.5 \pm 1.4$ & $26.0 \pm 1.6$ & $26.6 \pm 1.6$ \\
\hline & Week 4 & $31.7 \pm 1.7$ & $29.6 \pm 2.3$ & $29.3 \pm 1.8$ \\
\hline & Week 8 & $36.3 \pm 2.3$ & $32.2 \pm 2.5$ & $30.6 \pm 2.2$ \\
\hline \multirow[t]{4}{*}{ Heart rate (bpm) } & Baseline & $487.2 \pm 9.6$ & $503.9 \pm 12.8$ & $498.3 \pm 12.4$ \\
\hline & Week 1 & $525.8 \pm 16.7$ & $532.5 \pm 25.4$ & $526.2 \pm 13.0$ \\
\hline & Week 4 & $518.4 \pm 10.0$ & $484.9 \pm 11.2$ & $512.2 \pm 15.4$ \\
\hline & Week 8 & $518.3 \pm 13.3$ & $543.8 \pm 13.8$ & $513.5 \pm 15.9$ \\
\hline \multirow[t]{4}{*}{ Body weight (g) } & Baseline & $25.9 \pm 0.5$ & $24.9 \pm 0.4$ & $24.3 \pm 0.6$ \\
\hline & Week 1 & $25.5 \pm 0.5$ & $24.3 \pm 0.6$ & $24.4 \pm 0.6$ \\
\hline & Week 4 & $28.6 \pm 0.4$ & $27.8 \pm 0.4$ & $26.8 \pm 0.6$ \\
\hline & Week 8 & $30.5 \pm 0.5$ & $29.5 \pm 0.4$ & $29.6 \pm 0.6$ \\
\hline
\end{tabular}

${ }^{*} P<0.05,{ }^{* \star P} P<0.01,{ }^{* \star *} P<0.001$. WJMSC-EV-PLT: Wharton's Jelly-derived mesenchymal stem cell-extracellular vesicle-human platelet lysate; WJMSC-EV-FBS: Wharton's Jelly-derived mesenchymal stem cellextracellular vesicle-fetal bovine serum.

[Figure 7E, Table 2]. Although the slope of the end-systolic pressure-volume relationship (Ees) [Figure 7F, Table 2] was lower in the EV treated groups, the volume-axis intercept of ESPVR (Vo) [Figure 7G, Table 2] was substantially shifted leftward, indicating a positive effect on contractility. This was further supported by a marked increase in preload recruitable stroke work in WJMSC-EV-FBS vs. placebo [Figure 7H, Table 2]. Lastly, the slope of end-diastolic pressure-volume relationship significantly decreased in both EV groups, consistent with improved chamber compliance [Figure 7I, Table 2].

\section{Quantification of infarct size}

Masson's trichrome staining of myocardial histology sections was completed to compare infarct size at the 8-week endpoint [Figure 8A]. The quantification of infarct percentage compared to healthy myocardium allowed for the determination of cardiac injury. Interestingly, the infarct size in each EV group compared to the placebo control showed a significant decrease only in the WJMSC-EV-PLT group $(51.3 \% \pm 4.3 \%$ in WJMSC-EV-PLT $v s .63 .9 \% \pm 4.1 \%$ in placebo, $P<$ 0.05 , Figure $8 \mathrm{~B})$. 
Table 2. Hemodynamic PV loop measurements of cardiac function parameters

\begin{tabular}{|c|c|c|c|c|}
\hline & PV parameter & Placebo & WJMSC-EV-PLT & WJMSC-EV-FBS \\
\hline \multirow[t]{5}{*}{ Integrated performance } & $\mathrm{SW}\left(\mathrm{mmHg}^{\star} \mu \mathrm{L}\right)$ & $940.8 \pm 108.8$ & $1935 \pm 243.3^{\star \star}$ & $1712 \pm 213.6^{*}$ \\
\hline & $\mathrm{CO}(\mu \mathrm{L} / \mathrm{min})$ & $8619 \pm 811.0$ & $15079 \pm 1391^{\star \star}$ & $15578 \pm 1909^{\star \star}$ \\
\hline & $\mathrm{SV}(\mu \mathrm{L})$ & $16.7 \pm 1.6$ & $29.5 \pm 2.8^{\star \star}$ & $29.6 \pm 3.5^{\star \star}$ \\
\hline & $\mathrm{EF}(\%)$ & $10.9 \pm 1.0$ & $23.85 \pm 2.6^{\star \star}$ & $25.29 \pm 4.3^{\star \star}$ \\
\hline & $\mathrm{Ea} /$ Ees & $1.77 \pm 0.2$ & $2.67 \pm 0.4$ & $2.36 \pm 0.6$ \\
\hline \multirow[t]{2}{*}{ Afterload } & $\mathrm{ESP}(\mathrm{mmHg})$ & $81.1 \pm 3.9$ & $91.9 \pm 4.9$ & $81.6 \pm 4.5$ \\
\hline & $\mathrm{Ea}(\mathrm{mmHg} / \mathrm{mL})$ & $5.20 \pm 0.4$ & $3.36 \pm 0.3^{\star *}$ & $3.13 \pm 0.4^{\star \star \star}$ \\
\hline \multirow[t]{2}{*}{ Preload } & $\mathrm{EDP}(\mathrm{mmHg})$ & $13.5 \pm 1.6$ & $14.9 \pm 1.8$ & $14.6 \pm 2.3$ \\
\hline & $\operatorname{EDV}(\mu \mathrm{L})$ & $163.7 \pm 15.7$ & $132.3 \pm 9.6$ & $133.3 \pm 11.2$ \\
\hline \multirow[t]{5}{*}{ Contractility } & $\mathrm{dP} / \mathrm{dt} \max (\mathrm{mmHg} / \mathrm{s})$ & $5005 \pm 263.0$ & $5637 \pm 348.0$ & $5375 \pm 392.6$ \\
\hline & Ees $(\mathrm{mmHg} / \mathrm{mL})$ & $3.6 \pm 0.4$ & $2.0 \pm 0.5^{\star}$ & $1.7 \pm 0.3^{\star}$ \\
\hline & PRSW (mmHg) & $37.4 \pm 4.2$ & $47.6 \pm 5.9$ & $57.2 \pm 5.7^{\star}$ \\
\hline & $\mathrm{dP} / \mathrm{dt}$ max_EDV & $108.4 \pm 12.2$ & $99.4 \pm 21.4$ & $108.4 \pm 22.4$ \\
\hline & $V_{o}(\mu L)$ & $116.8 \pm 17.4$ & $65.1 \pm 10.0^{\star}$ & $60.5 \pm 9.7^{\star}$ \\
\hline \multirow[t]{5}{*}{ Lusitropy } & $\mathrm{dP} / \mathrm{dt} \min (\mathrm{mmHg} / \mathrm{s})$ & $-4113 \pm 258$ & $-4664 \pm 345$ & $-4472 \pm 431$ \\
\hline & Tau (ms) & $11.8 \pm 0.8$ & $11.4 \pm 0.7$ & $11.7 \pm 0.9$ \\
\hline & EDPVR & $0.07 \pm 0.02$ & $0.03 \pm 0.006^{\star \star}$ & $0.03 \pm 0.004^{\star}$ \\
\hline & $\operatorname{ESV}(\mu \mathrm{L})$ & $142.7 \pm 16.6$ & $104.9 \pm 12.0$ & $107.4 \pm 12.8$ \\
\hline & Heart rate (bpm) & $514.0 \pm 4.5$ & $517.2 \pm 11.6$ & $523.9 \pm 6.2$ \\
\hline
\end{tabular}

${ }^{\star} P<0.05,{ }^{* \star} P<0.01,{ }^{* \star *} P<0.001$. PV: Pressure-volume; WJMSC-EV-PLT: Wharton's Jelly-derived mesenchymal stem cell-extracellular vesiclehuman platelet lysate; WJMSC-EV-FBS: Wharton's Jelly-derived mesenchymal stem cell-extracellular vesicle-fetal bovine serum; SW: stroke work; CO: cardiac output; SV: stroke volume; EF: ejection fraction; Ea/Ees: arterial-ventricular coupling ratio; ESP: end-systolic pressure; Ea: arterial elastance; EDP: end-diastolic pressure; EDV: end-diastolic volume; Ees: slope of end-systolic pressure-volume relationship; PRSW: preload recruitable stroke work; Vo: volume-axis intercept of ESPVR; EDPVR: end-diastolic pressure-volume relationship; ESV: end-systolic volume.

\section{DISCUSSION}

The translation of EV therapy has been challenged by the need for large-scale production of EVs in an efficient and reproducible manner ${ }^{[15]}$. Typical cell culture expansion of MSCs in flasks can produce sufficient EV numbers for small animal experiments. However, for translation of EV doses to humans, EV production would need to expand significantly to produce sufficient numbers of EVs for multiple patients or potentially multiple doses for a single patient. The Quantum bioreactor is established as an effective tool for MSC expansion and has recently been adopted by several cell production faculties to achieve their large-scale production goals ${ }^{[27]}$.

The Quantum bioreactor contains a 3-dimensional hollow-fiber closed bioreactor with approximately 11,500 hollow fibers that provide $2.1 \mathrm{~m}^{2}$ of cell growth surface area, which is roughly equivalent to $120 \mathrm{~T}$ flasks $\left(175 \mathrm{~mm}^{2}\right)$. Inward and outward media flow lines facilitate the supply of fresh media to cells and the collection of EV-conditioned media. Our study has further demonstrated that the Quantum bioreactor is an effective tool for large-scale WJMSC expansion and subsequent EV isolation. In this study, we demonstrate the superiority of PLT media expansion in the Quantum bioreactor for high cell yields compared to FBS supplemented expansion. Furthermore, those PLT expanded cells tended to produce a slightly greater yield of EV particles compared to the FBS expanded counterpart. Although this result was not significant, the higher cell expansion and reproducibility of EV particle yield suggest PLT as the optimal expansion media choice for EV harvest in serum-free media. Our EV production methodology included sequential centrifugation and precipitation at 100,000 $\mathrm{xg}$ to concentrate the fraction of small EVs. Nanoparticle analysis and flow cytometry confirmed the presence of small EVs. These products had a particle mode size 

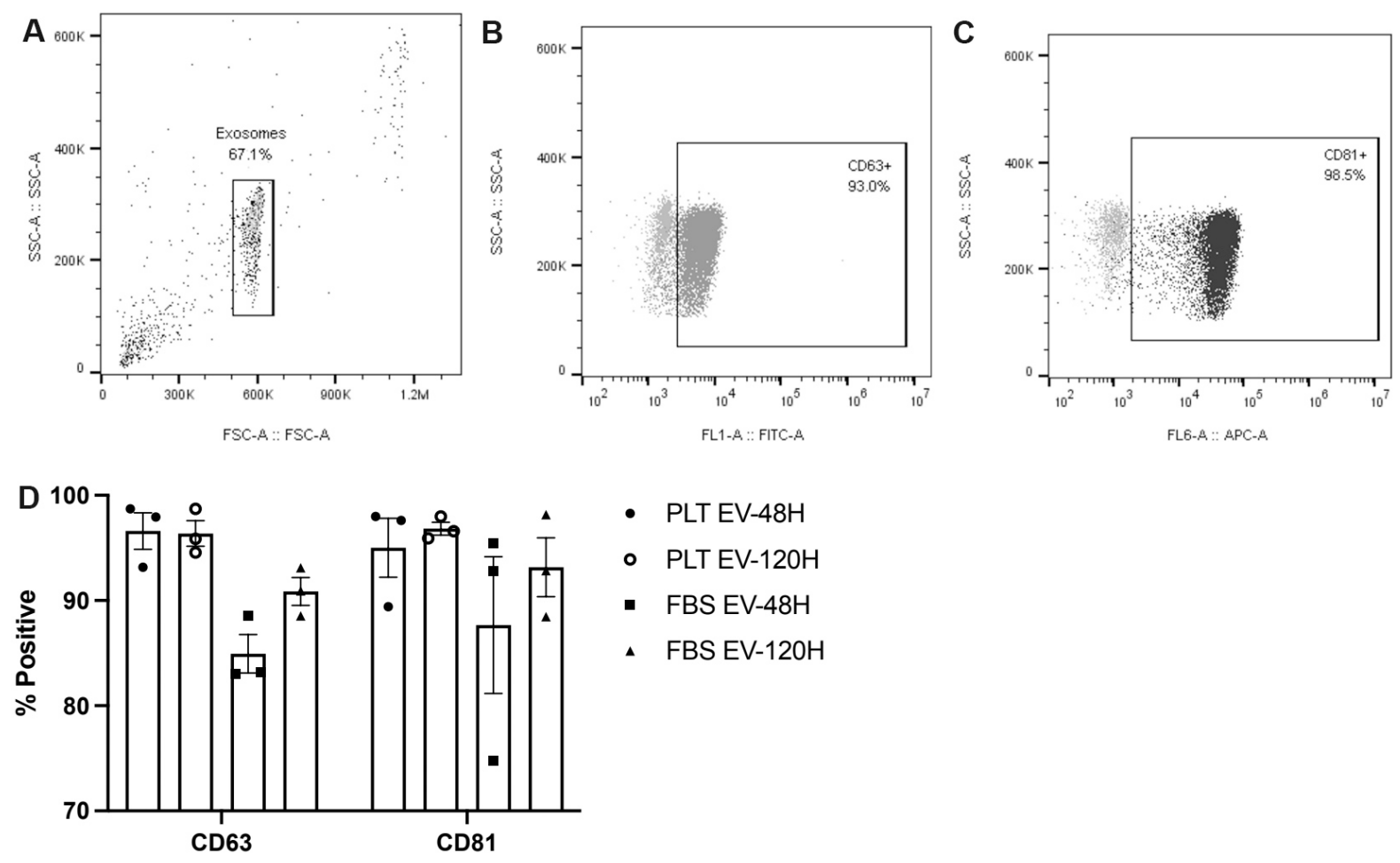

Figure 2. Flow cytometry analysis of the EV products. (A) Representative scatter plots showing the analyzed EV population. (B) Positive expression of FITC+/CD63+ EVs (dark gray) compared to isotype control (light gray). (C) Positive expression of APC+/CD81+ EVs (black) compared to isotype control (light gray). (D) The calculated CD63 and CD81 positive expression of the EV products collected at the 48 and 120 h collection time points. EVs: Extracellular vesicles; PLT: human platelet lysate; FBS: fetal bovine serum.

below $150 \mathrm{~nm}$ and were positive for CD63 and CD81 ${ }^{[28]}$.

The suitability of the Quantum bioreactor as a large-scale platform for EV production is further demonstrated by the total EV production yield and the production of clinically relevant size doses. Although optimized EV doses have yet to be established and proven in the clinic, small and large animal studies have established ranges of effective doses to be considered for clinical use. Reported doses tested in mice ranged from $1 \times 10^{9}$ to $3 \times 10^{10}$ total particles, whereas pig models have EV doses ranging from $1 \times 10^{12}$ to $3 \times 10^{12}$ total particles per dose $\mathrm{e}^{[29,30]}$. With the assumption that a human dose would be similar to that of a large animal, our methodology was able to produce a minimum of 3-10 clinical doses of EV from a single 2week long manufacturing run. This methodology easily meets the need of initial phase I and II clinical trials designed to test the safety and efficacy of MSC-derived EVs.

While it is well established that PLT is an effective alternative to FBS for WJMSC expansion without altering the therapeutic properties, it is unknown if expansion media modulates EV cargo. EV cargo, particularly miRNA, plays a vital role in cardio-protection and cardiac regeneration when transferred to damaged cells ${ }^{[31,32]}$. Therefore, we identified the EV miRNA cargo produced from the FBS and PLT methods. We found few variations in miRNA profiles with regard to miRNA content. Most of the miRNA content was shared between the FBS and PLT products, and bioinformatics analysis found multiple MI-associated gene targeting in similarly expressed miRNA. The identified uniquely expressed miRNAs all fell under $1075 \mathrm{read}$ counts, with the majority under 400 read counts. Therefore, the uniquely targeted gene pathways may not provide a significant therapeutic advantage. 


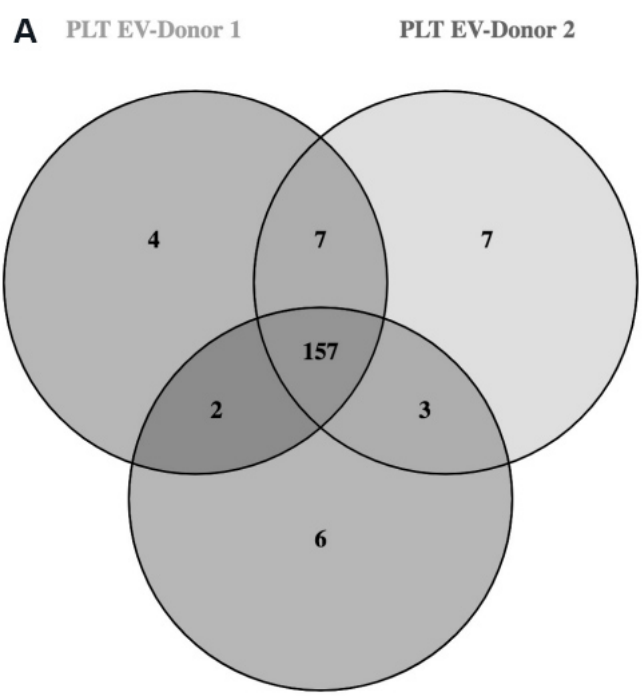

PLT EV-Donor 3

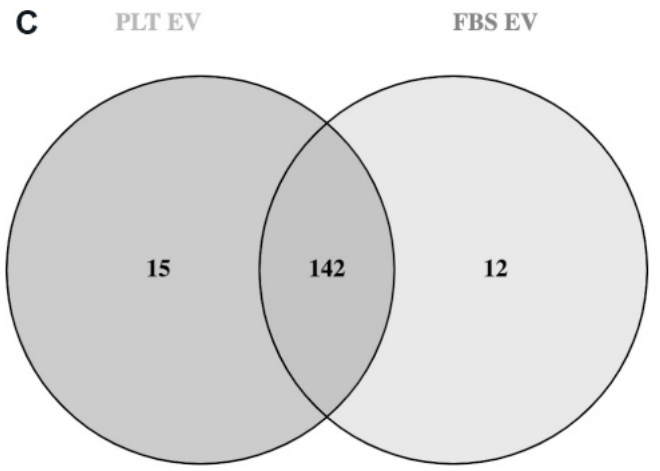

B FBS EV-Donor $1 \quad$ FBS EV-Donor 2

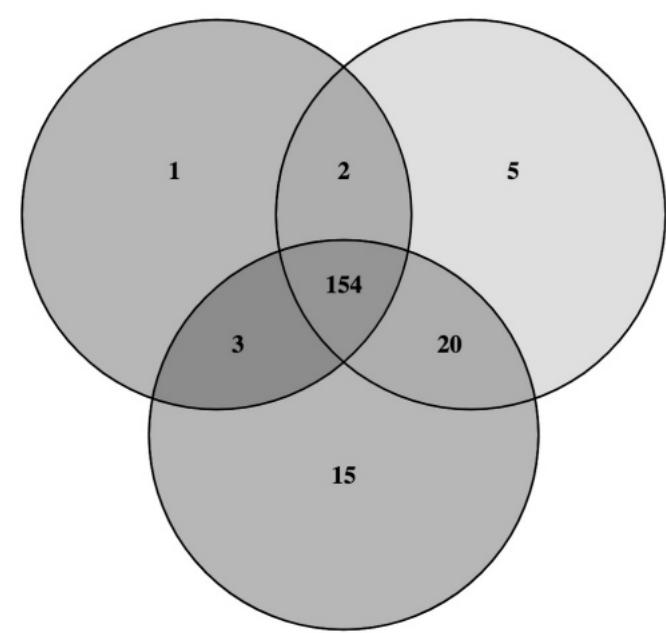

FBS EV-Donor 3

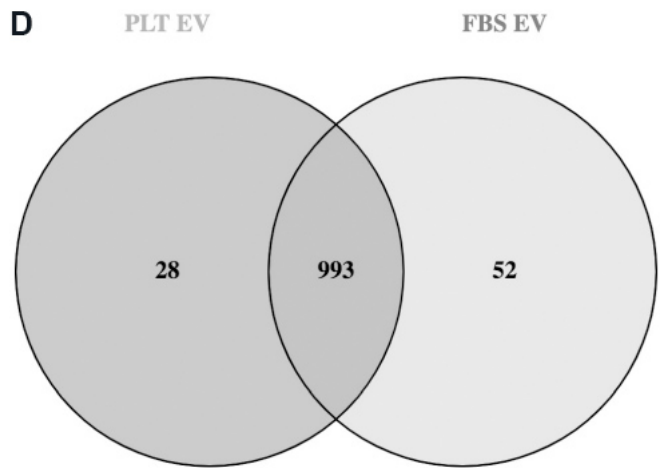

Figure 3. Identification and differential expression analysis of EV miRNA cargo. (A) The identification of commonly expressed miRNA between 3 different PLT-EV products manufactured from 3 different donor materials. (B) The identification of commonly expressed miRNA between 3 different FBS-EV products manufactured from 3 different donor materials. (C) The identification of the commonly expressed miRNA and the unique miRNA in the PLT and FBS groups. (D) The identification of common and unique miRNA gene targets. EVs: Extracellular vesicles; PLT: human platelet lysate; FBS: fetal bovine serum.

Following the large-scale manufacturing of the WJMSC EV products, we proceeded to test their therapeutic potential in our established model of MI. We sought to investigate if these products could rescue cardiac function after an established myocardial infarction injury when delivered in a systemic manner. Due to the large EV product yield, we were able to explore this proof-of-concept experiment by delivering multiple EV administrations throughout the study time course. Since the intrajugular injection is an indirect delivery route of EVs to target the injury site, we explored a repeated administration strategy to determine a potential therapeutic effect. We note that additional experiments may be required to determine the optimal dosing strategy.

We started administering the EV treatment 1 week after the established MI injury via intra-jugular injection. Subsequent injections were completed every week for 6 weeks, and an analysis of the animals was performed at 8 weeks post-MI to determine long-term changes to cardiac function from the repeated administration. It is important to note that no reported adverse side effects were noticed with the multiple injections. With our current treatment strategy, both echocardiography and hemodynamic analysis 


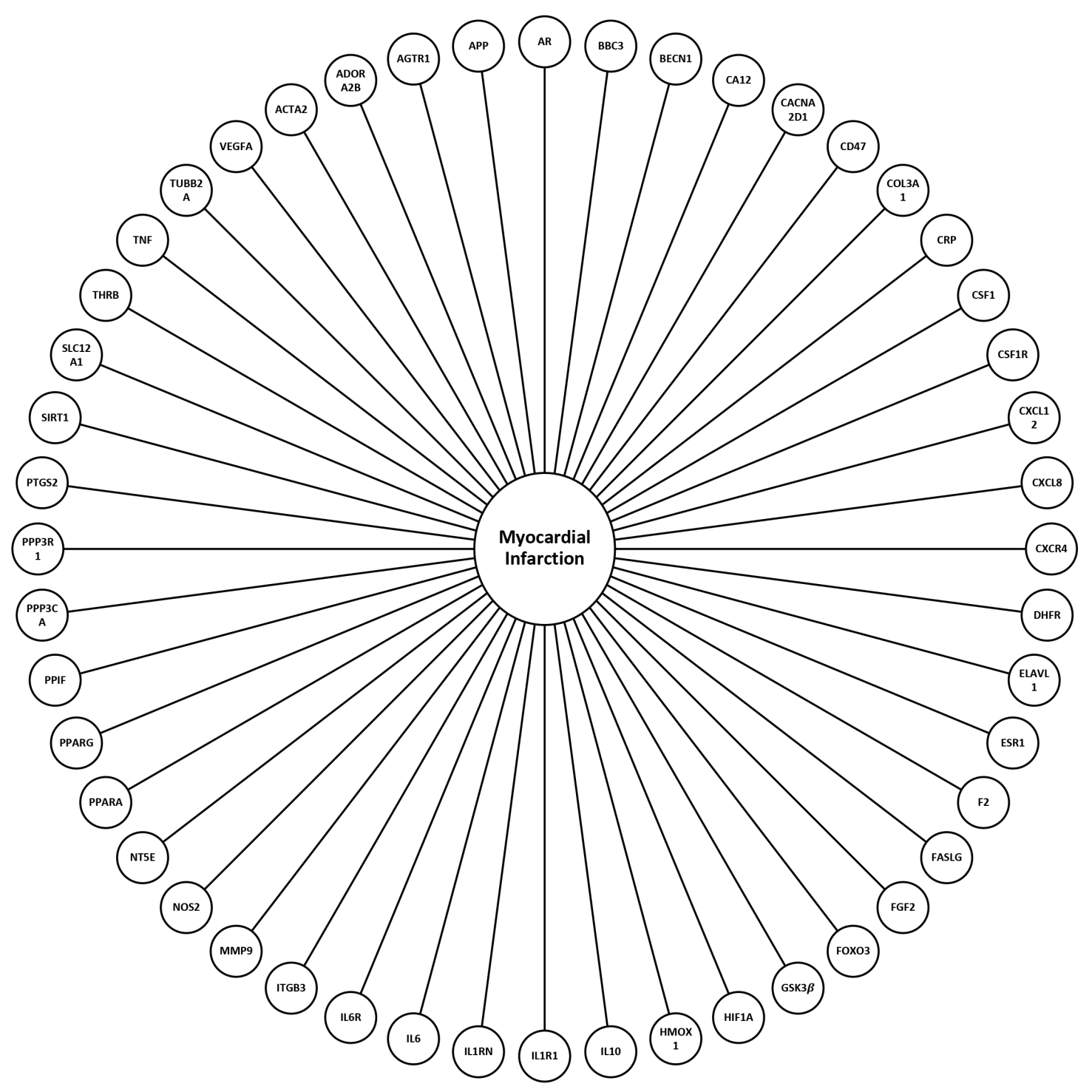

Figure 4. Bioinformatics analysis of MI gene targets. WJMSC EV miRNA gene targets associated with MI. MI: Myocardial infarction; WJMSC: Wharton's Jelly-derived mesenchymal stem cell; EV: extracellular vesicle.

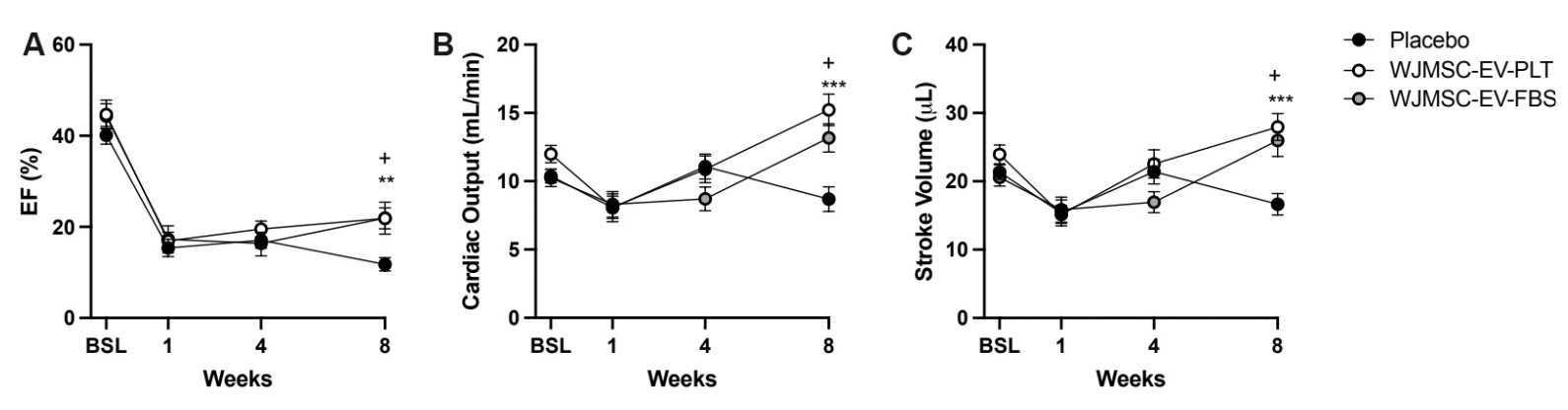

Figure 5. Echocardiography measurements of cardiac function parameters. (A) Ejection fraction percentage at each time point. (B) Cardiac output at each time point. (C) Stroke volume at each time point. (D) Fractional shortening at each time point. Baseline $n=10-13$ in all groups, week 1, 4, and $8 n=14$. ${ }^{\star \star} P<0.01,{ }^{\star \star \star} * P<0.001,{ }^{+} P<0.05$. EF: Ejection fraction; WJMSC: Wharton's Jelly-derived mesenchymal stem cell; EV: extracellular vesicle; PLT: human platelet lysate; FBS: fetal bovine serum. 


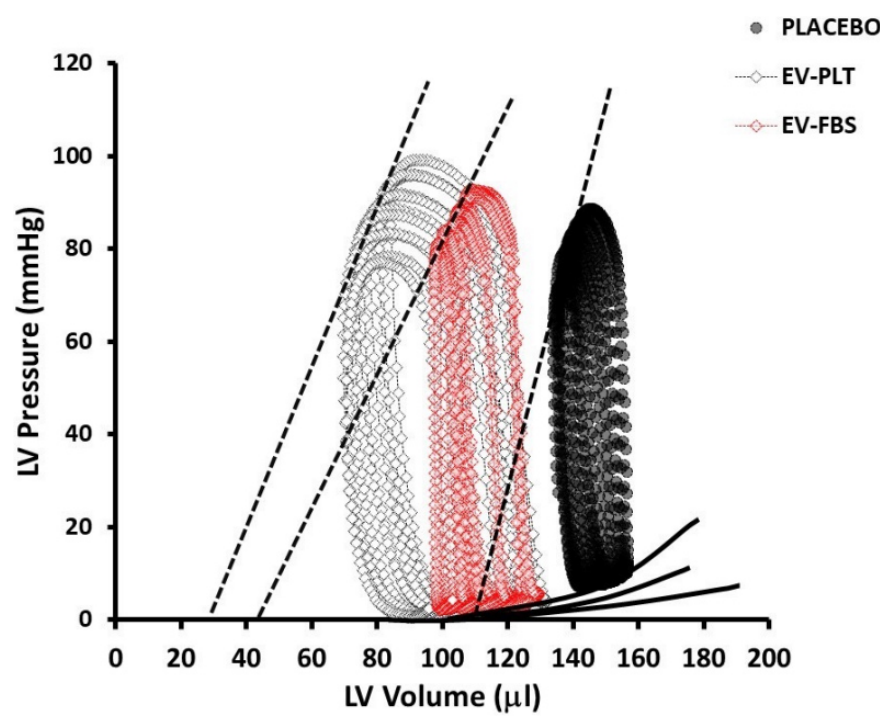

Figure 6. Hemodynamic PV loop measurements. Representative PV loops from each experimental group. EV: Extracellular vesicle; PLT: human platelet lysate; FBS: fetal bovine serum; LV: left ventricle; PV: pressure-volume.

A

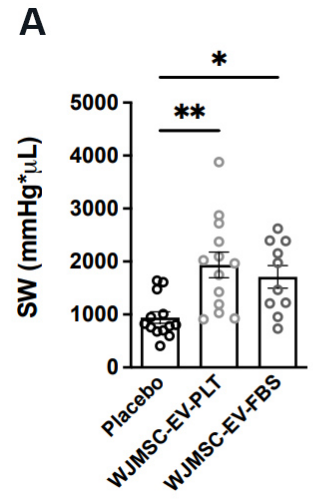

$\mathbf{F}$

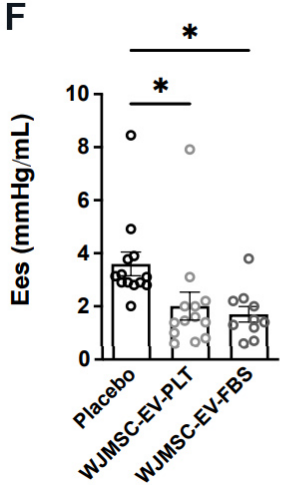

B

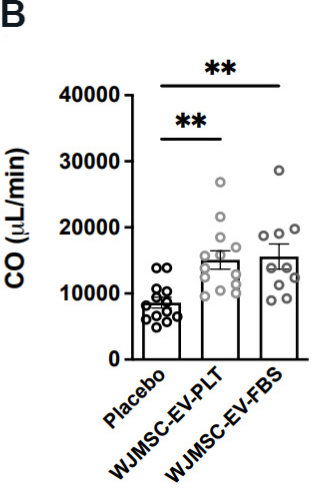

G

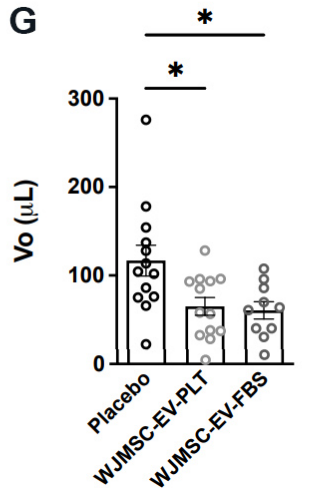

C

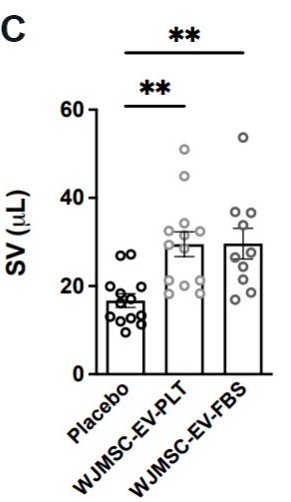

H

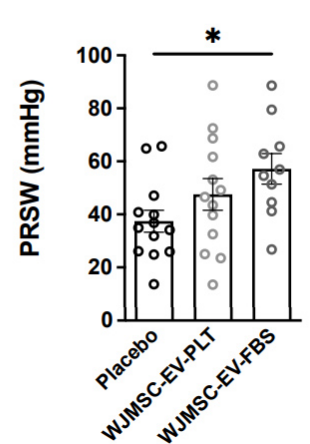

D
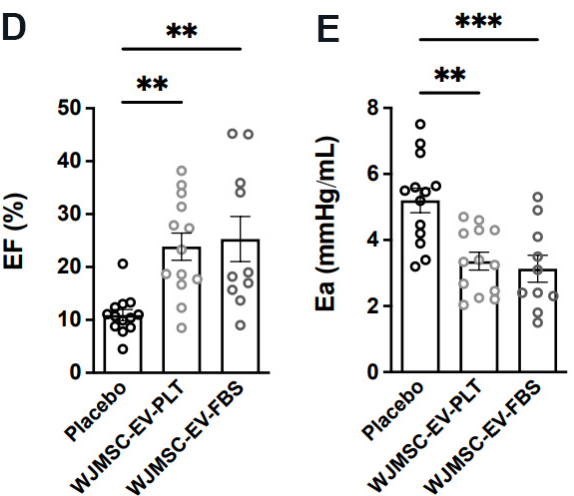

I

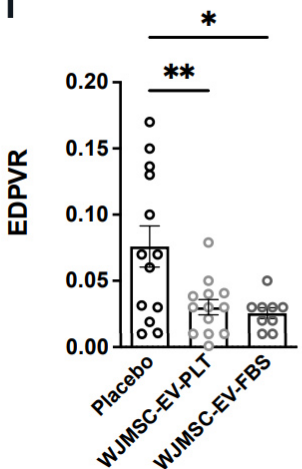

Figure 7. Hemodynamic PV loop measurements of cardiac function parameters. PV loop analysis of: (A) stroke work (SW); (B) cardiac output (CO); (C) stroke volume (SV); (D) ejection fraction (EF); (E) arterial elastance (Ea); (F) slope of end-systolic pressure-volume relationship (Ees); (G) volume-axis intercept of ESPVR (Vo); (H) preload recruitable stroke work (PRSW); and (I) slope of end-diastolic pressure-volume relationship (EDPVR). Placebo $n=13$, WJMSC-EV-PLT $n=13$, WJMSC-EV-FBS $n=10 .{ }^{\star} P<0.05,{ }^{\star \star} P<0.01,{ }^{\star \star \star} P<$ 0.001. WJMSC: Wharton's Jelly-derived mesenchymal stem cell; EV: extracellular vesicle; PLT: human platelet lysate; FBS: fetal bovine serum. 


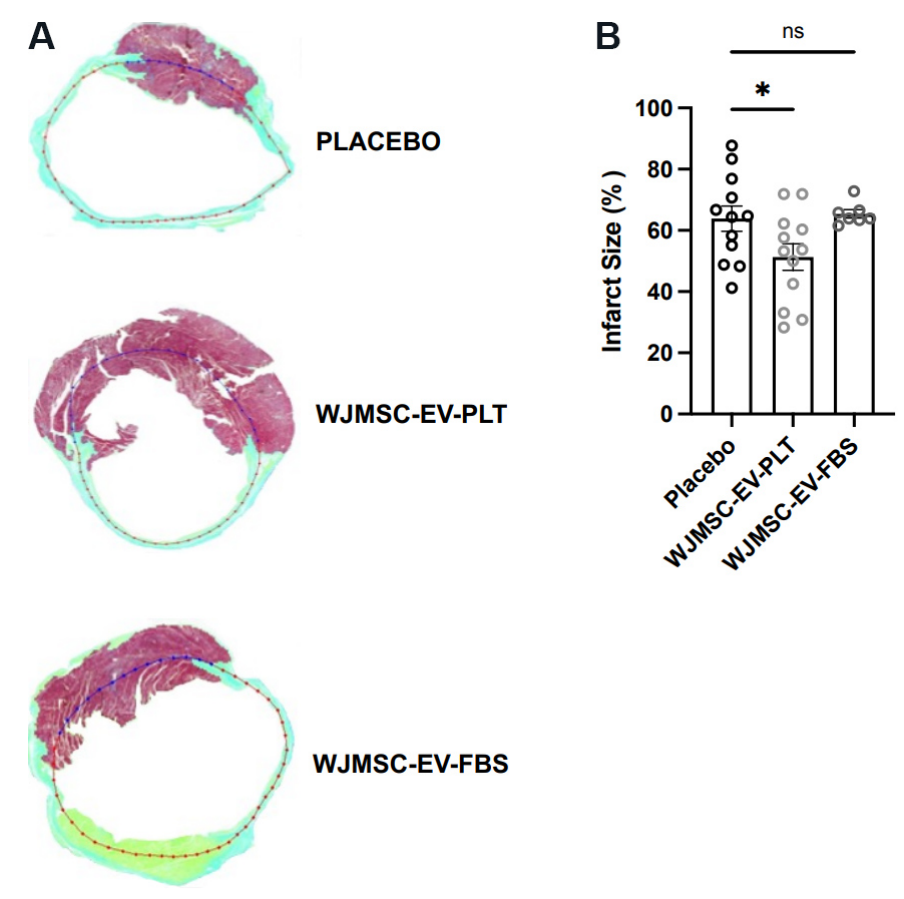

Figure 8. Myocardial infarction size. (A) Representative Masson's trichrome staining of heart sections at the midventricular level of each treatment group. (B) Comparative quantification of infarct size percentage. Placebo $n=12$, WJMSC-EV-PLT $n=12$, WJMSC-EVFBS $n=7 .{ }^{*} P<0.05$. WJMSC: Wharton's Jelly-derived mesenchymal stem cell; EV: extracellular vesicle; PLT: human platelet lysate; FBS: fetal bovine serum.

confirmed EV-mediated improvements in cardiac function. Important parameters such as integrated performance, afterload, contractility, and lusitropy were all found to be significantly improved by the EV treatment. Surprisingly, Ees was reduced in both treated groups; however, this finding occurred in the presence of a substantial leftward shift in Vo indicated an extreme degree of remodeling, a situation where changes in afterload can affect Ees ${ }^{[33-35]}$. In addition, as the ventricular pressure gets down to $\sim 50-60 \mathrm{mmHg}$, the heart perfusion is limited, resulting in a curvilinear instead of linear response $e^{[36,37]}$. Changes in Ees can also reflect changes in cardiac morphometry such as hypertrophy and fibrosis; thus, it can misrepresent contractile function ${ }^{[3,393}$. On the other hand, Vo provides a good LV contractility index due to less sensitivity to large changes in afterload conditions ${ }^{[35]}$. Accordingly, a higher Vo in the placebo group reflects lower contractility.

Aside from function, a decrease in cardiac infarct size was observed in the WJMSC-EV-PLT group, indicating a possible anti-fibrotic effect that may have contributed to an improvement in cardiac function. Surprisingly, this anti-fibrotic effect was not observed in the WJMSC-EV-FBS group. We hypothesize that the lack of improvement in cardiac fibrosis with the FBS-EVs may be related to limited retention of EVs in the myocardium compared to the PLT-EVs, which may have led to differences in the reduction in the infarction microenvironment. Instead, both FBS-EV and PLT-EV products improved cardiac function, perhaps due to a systemic effect that was achieved through the modulation of remote myocardial remodeling. The coronary microvasculature undergoes increased vascular permeability and endothelial dysfunction associated with $\mathrm{MI}^{[40]}$. Accordingly, effective cardioprotective effects include not only infarct size reduction but also positive effects on coronary microcirculation, inflammation, and vascular integrity ${ }^{[4]}$. Therefore, future studies to define the contribution of coronary microcirculation and extend the time-frame follow-up of cardiac performance are warranted to elucidate the therapeutic efficacy of EVs. Nonetheless, the improvements in cardiac function along with reduced infarction size with the PLT-EV 
treatment make this product an important candidate for therapeutic trials.

Previous publications have suggested several mechanisms of action for EV-induced cardiac regeneration ${ }^{[42]}$, including the transfer of miRNA EV cargo to restore signaling pathways that promote angiogenesis, macrophage polarization, and decreased fibrosis ${ }^{[43-46]}$. With the vast targeting of miRNAs, it is likely that EVinduced repair is not limited to a single mechanism but is, in fact, a coordinated effect induced by multiple pathways.

In conclusion, our work demonstrates the feasibility of using large-scale expansion systems to produce high quantities of potent EVs for regenerative therapies. This study serves as a starting point for EV translation into clinical research via a validated manufacturing procedure and demonstrated proof-of-concept animal study. Furthermore, these results have potential clinical implications for the treatment of MI and other cardiac injuries.

\section{DECLARATIONS}

\section{Authors' contributions}

Designed the study and performed experiments: Bellio MA, Kanashiro-Takeuchi RM, Takeuchi L, Kulandavelu S, Lee YS, Balkan W, Young KC, Hare JM, Khan A

Guided the interpretation of the results: Bellio MA, Kanashiro-Takeuchi RM, Hare JM

Prepared the manuscript: Bellio MA, Kanashiro-Takeuchi RM, Balkan W, Hare JM

\section{Availability of data and materials}

The source data used to make all the conclusions is presented within this article and supplementary tables. RNA sequencing data used for bioinformatics is provided within the supplement tables. Further inquiries can be directed to the corresponding author.

\section{Financial support and sponsorship}

This work was supported by NHLBI-PACT HHSN268201600012I/75N92018R0050, NHLBI-PACT PCT0012-01, National Institutes of Health grants R01 HL107110 and R01 HL137355, The Marcus Foundation.

\section{Conflicts of interest}

Hare JM reported having a patent for cardiac cell-based therapy. He holds equity in Vestion Inc. and maintains a professional relationship with Vestion Inc. as a consultant and member of the Board of Directors and Scientific Advisory Board. Hare JM is the Chief Scientific Officer, a compensated consultant and advisory board member for Longeveron, and holds equity in Longeveron. Hare JM is also the coinventor of intellectual property licensed to Longeveron. Longeveron LLC and Vestion Inc. did not participate in funding this work. Hare JM's relationships are disclosed to the University of Miami and a management plan is in place. Khan A discloses a relationship with AssureImmune Cord Blood Bank, AX Biotech, and Aceso Therapeutics that includes equity. The other authors declared that there are no conflicts of interest. The University of Miami is an equity owner in Longeveron, which has licensed intellectual property from the University of Miami.

\section{Ethical approval and consent to participate}

This study protocol and written informed consent were reviewed and approved by the Institutional Review Board of the University of Miami (IRB number 20100986). 


\section{Consent for publication}

Not applicable.

\section{Copyright}

(c) The Author(s) 2022.

\section{REFERENCES}

1. North BJ, Sinclair DA. The intersection between aging and cardiovascular disease. Circ Res 2012;110:1097-108. DOI PubMed PMC

2. Bagno L, Hatzistergos KE, Balkan W, Hare JM. Mesenchymal stem cell-based therapy for cardiovascular disease: progress and challenges. Mol Ther 2018;26:1610-23. DOI PubMed PMC

3. Schulman IH, Hare JM. Mesenchymal stromal cells as a therapeutic intervention. In: Valarmathi MT, editor. Stromal cells - structure, function, and therapeutic implications. IntechOpen; 2019. DOI

4. Kanelidis AJ, Premer C, Lopez J, Balkan W, Hare JM. Route of delivery modulates the efficacy of mesenchymal stem cell therapy for myocardial infarction: a meta-analysis of preclinical studies and clinical trials. Circ Res 2017;120:1139-50. DOI PubMed PMC

5. Hare JM, Fishman JE, Gerstenblith G, et al. Comparison of allogeneic vs autologous bone marrow-derived mesenchymal stem cells delivered by transendocardial injection in patients with ischemic cardiomyopathy: the POSEIDON randomized trial. JAMA 2012;308:2369-79. DOI PubMed PMC

6. Hare JM, DiFede DL, Rieger AC, et al. Randomized comparison of allogeneic versus autologous mesenchymal stem cells for nonischemic dilated cardiomyopathy: POSEIDON-DCM trial. J Am Coll Cardiol 2017;69:526-37. DOI PubMed PMC

7. Florea V, Rieger AC, DiFede DL, et al. Dose comparison study of allogeneic mesenchymal stem cells in patients with ischemic cardiomyopathy (The TRIDENT Study). Circ Res 2017;121:1279-90. DOI PubMed

8. Rani S, Ryan AE, Griffin MD, Ritter T. Mesenchymal stem cell-derived extracellular vesicles: toward cell-free therapeutic applications. Mol Ther 2015;23:812-23. DOI PubMed PMC

9. Mendt M, Rezvani K, Shpall E. Mesenchymal stem cell-derived exosomes for clinical use. Bone Marrow Transplant 2019;54:789-92. DOI PubMed

10. Burke J, Kolhe R, Hunter M, Isales C, Hamrick M, Fulzele S. Stem cell-derived exosomes: a potential alternative therapeutic agent in orthopaedics. Stem Cells Int 2016;2016:5802529. DOI PubMed PMC

11. Elahi FM, Farwell DG, Nolta JA, Anderson JD. Preclinical translation of exosomes derived from mesenchymal stem/stromal cells. Stem Cells 2020;38:15-21. DOI PubMed PMC

12. H Rashed M, Bayraktar E, K Helal G, et al. Exosomes: from garbage bins to promising therapeutic targets. Int J Mol Sci 2017;18:538. DOI PubMed PMC

13. Ibrahim A, Marbán E. Exosomes: fundamental biology and roles in cardiovascular physiology. Annu Rev Physiol 2016;78:67-83. DOI PubMed PMC

14. Tan L, Wu H, Liu Y, Zhao M, Li D, Lu Q. Recent advances of exosomes in immune modulation and autoimmune diseases. Autoimmunity 2016;49:357-65. DOI PubMed

15. Willis GR, Kourembanas S, Mitsialis SA. Toward exosome-based therapeutics: isolation, heterogeneity, and fit-for-purpose potency. Front Cardiovasc Med 2017;4:63. DOI PubMed PMC

16. Kishore R, Khan M. More Than tiny sacks: stem cell exosomes as cell-free modality for cardiac repair. Circ Res 2016;118:330-43. DOI PubMed PMC

17. Colao IL, Corteling R, Bracewell D, Wall I. Manufacturing exosomes: a promising therapeutic platform. Trends Mol Med 2018;24:242-56. DOI PubMed

18. Kandoi S, L PK, Patra B, et al. Evaluation of platelet lysate as a substitute for FBS in explant and enzymatic isolation methods of human umbilical cord MSCs. Sci Rep 2018;8:12439. DOI PubMed PMC

19. Becherucci V, Piccini L, Casamassima S, et al. Human platelet lysate in mesenchymal stromal cell expansion according to a GMP grade protocol: a cell factory experience. Stem Cell Res Ther 2018;9:124. DOI PubMed PMC

20. Barile L, Moccetti T, Marbán E, Vassalli G. Roles of exosomes in cardioprotection. Eur Heart J 2017;38:1372-9. DOI PubMed

21. Dougherty JA, Mergaye M, Kumar N, Chen CA, Angelos MG, Khan M. Potential role of exosomes in mending a broken heart: nanoshuttles propelling future clinical therapeutics forward. Stem Cells Int 2017;2017:5785436. DOI PubMed PMC

22. Yang L, Zhu J, Zhang C, et al. Stem cell-derived extracellular vesicles for myocardial infarction: a meta-analysis of controlled animal studies. Aging (Albany NY) 2019;11:1129-50. DOI PubMed PMC

23. Hatzistergos KE, Paulino EC, Dulce RA, et al. S-nitrosoglutathione reductase deficiency enhances the proliferative expansion of adult heart progenitors and myocytes post myocardial infarction. J Am Heart Assoc 2015;4:e001974. DOI PubMed PMC

24. Parvatiyar MS, Brownstein AJ, Kanashiro-Takeuchi RM, et al. Stabilization of the cardiac sarcolemma by sarcospan rescues DMDassociated cardiomyopathy. JCI Insight 2019;5:123855. DOI PubMed PMC

25. Nascimento DS, Valente M, Esteves T, et al. MIQuant--semi-automation of infarct size assessment in models of cardiac ischemic injury. PLoS One 2011;6:e25045. DOI PubMed PMC

26. Takagawa J, Zhang Y, Wong ML, et al. Myocardial infarct size measurement in the mouse chronic infarction model: comparison of area- and length-based approaches. J Appl Physiol (1985) 2007;102:2104-11. DOI PubMed PMC

27. Hanley PJ, Mei Z, Durett AG, et al. Efficient manufacturing of therapeutic mesenchymal stromal cells with the use of the Quantum 
Cell Expansion System. Cytotherapy 2014;16:1048-58. DOI PubMed PMC

28. Doyle LM, Wang MZ. Overview of extracellular vesicles, their origin, composition, purpose, and methods for exosome isolation and analysis. Cells 2019;8:727. DOI PubMed PMC

29. Gallet R, Dawkins J, Valle J, et al. Exosomes secreted by cardiosphere-derived cells reduce scarring, attenuate adverse remodelling, and improve function in acute and chronic porcine myocardial infarction. Eur Heart J 2017;38:201-11. DOI PubMed PMC

30. Williams AM, Bhatti UF, Brown JF, et al. Early single-dose treatment with exosomes provides neuroprotection and improves bloodbrain barrier integrity in swine model of traumatic brain injury and hemorrhagic shock. J Trauma Acute Care Surg 2020;88:207-18. DOI PubMed

31. Jung JH, Ikeda G, Tada Y, et al. miR-106a-363 cluster in extracellular vesicles promotes endogenous myocardial repair via Notch3 pathway in ischemic heart injury. Basic Res Cardiol 2021;116:19. DOI PubMed

32. Moghaddam AS, Afshari JT, Esmaeili SA, Saburi E, Joneidi Z, Momtazi-Borojeni AA. Cardioprotective microRNAs: lessons from stem cell-derived exosomal microRNAs to treat cardiovascular disease. Atherosclerosis 2019;285:1-9. DOI PubMed

33. Burkhoff D. Pressure-volume loops in clinical research: a contemporary view. J Am Coll Cardiol 2013;62:1173-6. DOI PubMed

34. Burkhoff D, Tyberg JV. Why does pulmonary venous pressure rise after onset of LV dysfunction: a theoretical analysis. Am J Physiol 1993;265:H1819-28. DOI PubMed

35. Blaudszun G, Morel DR. Relevance of the volume-axis intercept, V0, compared with the slope of end-systolic pressure-volume relationship in response to large variations in inotropy and afterload in rats. Exp Physiol 2011;96:1179-95. DOI PubMed

36. Burkhoff D, Sugiura S, Yue DT, Sagawa K. Contractility-dependent curvilinearity of end-systolic pressure-volume relations. Am J Physiol 1987;252:H1218-27. DOI PubMed

37. Claessens TE, Georgakopoulos D, Afanasyeva M, et al. Nonlinear isochrones in murine left ventricular pressure-volume loops: how well does the time-varying elastance concept hold? Am J Physiol Heart Circ Physiol 2006;290:H1474-83. DOI PubMed

38. Sharp TE 3rd, Schena GJ, Hobby AR, et al. Cortical bone stem cell therapy preserves cardiac structure and function after myocardial infarction. Circ Res 2017;121:1263-78. DOI PubMed PMC

39. Pacher P, Nagayama T, Mukhopadhyay P, Bátkai S, Kass DA. Measurement of cardiac function using pressure-volume conductance catheter technique in mice and rats. Nat Protoc 2008;3:1422-34. DOI PubMed PMC

40. Heusch G. The coronary circulation as a target of cardioprotection. Circ Res 2016;118:1643-58. DOI PubMed

41. Heusch G. Critical issues for the translation of cardioprotection. Circ Res 2017;120:1477-86. DOI PubMed

42. Qiu G, Zheng G, Ge M, et al. Mesenchymal stem cell-derived extracellular vesicles affect disease outcomes via transfer of microRNAs. Stem Cell Res Ther 2018;9:320. DOI PubMed PMC

43. Ferguson SW, Wang J, Lee CJ, et al. The microRNA regulatory landscape of MSC-derived exosomes: a systems view. Sci Rep 2018;8:1419. DOI PubMed PMC

44. Zhao J, Li X, Hu J, et al. Mesenchymal stromal cell-derived exosomes attenuate myocardial ischaemia-reperfusion injury through miR-182-regulated macrophage polarization. Cardiovasc Res 2019;115:1205-16. DOI PubMed PMC

45. Luther KM, Haar L, McGuinness M, et al. Exosomal miR-21a-5p mediates cardioprotection by mesenchymal stem cells. J Mol Cell Cardiol 2018;119:125-37. DOI PubMed

46. Xiao C, Wang $\mathrm{K}, \mathrm{Xu} \mathrm{Y}$, et al. Transplanted mesenchymal stem cells reduce autophagic flux in infarcted hearts via the exosomal transfer of miR-125b. Circ Res 2018;123:564-78. DOI PubMed 